\title{
SSRI-associated bruxism
}

\section{A systematic review of published case reports}

Andrew R. Garrett, DO, MPH, MS and Jason S. Hawley, MD

Neurology: Clinical Practice April 2018 vol. 8 no. 2 135-141 doi:10.1212/CPJ.0000000000000433
Correspondence

Dr. Garrett

andrew.r.garrett11.mil@mail.mil

\begin{abstract}
Purpose of review

Antidepressant-associated movement disorders are a welldescribed phenomenon. However, antidepressant-associated bruxism, jaw pain, or jaw spasm, while reported in dental literature, is less commonly recognized among neurologists. We summarize the clinical features and treatment of antidepressantassociated bruxism and associated jaw pain through a systematic review of case reports.
\end{abstract}

\section{Recent findings}

Antidepressant-associated bruxism may occur in pediatric and adult

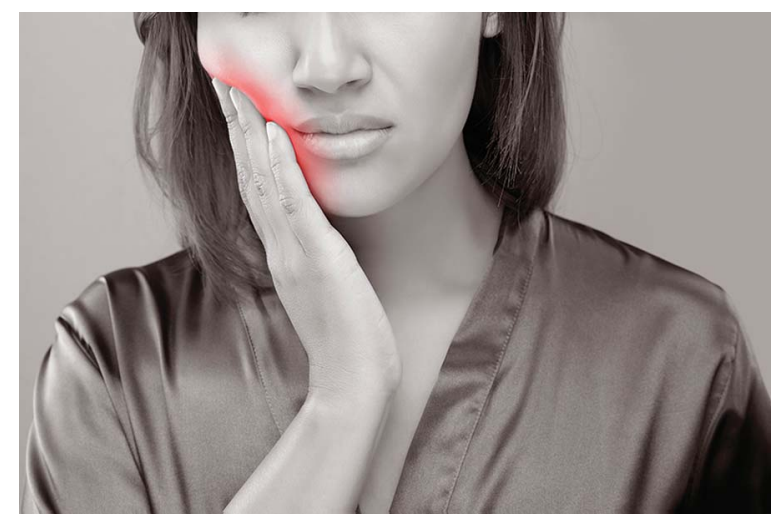
patients, most commonly among female patients. Patients may develop symptoms with shortterm and long-term antidepressant use. Fluoxetine, sertraline, and venlafaxine were the most commonly reported offending agents. Symptoms may begin within 3-4 weeks of medication initiation and may resolve within 3-4 weeks of drug discontinuation, addition of buspirone, or substitution with another pharmacologic agent. The incidence of this phenomenon is unknown.

\section{Summary}

Bruxism associated with antidepressant use is an underrecognized phenomenon among neurologists, and may be treated with the addition of buspirone, dose modification, or medication discontinuation.

Bruxism is a common stereotyped movement disorder characterized by repetitive clenching of the jaw and grinding of the teeth. ${ }^{1}$ Sleep bruxism is associated with sleep arousal, and is characterized by lateral teeth grinding. Patients often complain of jaw pain and trismus upon awakening, and bed partners often witness or hear grinding during nighttime hours. Bruxism can also occur during wakefulness, and is often associated with stress. Severe cases of bruxism can result in extreme tooth wear or tooth fracture. ${ }^{2}$ Jaw pain is common, along with headaches, facial pain, and sleep disorders. Despite its comorbid association with neurologic disorders such as migraine, facial pain, and sleep dysregulation, much of the literature on bruxism is outside of general neurology in sleep medicine and dentistry. The causes of bruxism are poorly understood, and often related to emotional stress. The understanding of the cause of the condition is complex, thought to be entirely involuntary or as a habit/ behavior described as parafunctional. Despite the notion of bruxism being a behavioral phenomenon, there are several case reports and series describing a possible relationship between bruxism and serotonergic antidepressants, including selective serotonin reuptake inhibitors (SSRIs) and serotonin-norepinephrine reuptake inhibitors (SNRIs). Metaanalyses of these cases are lacking, and there are few data on the incidence, clinical

Department of Neurology, Walter Reed National Military Medical Center, Bethesda, MD.

Funding information and disclosures are provided at the end of the article. Full disclosure form information provided by the authors is available with the full text of this article at Neurology.org/cp.

The views expressed in manuscript are those of the authors alone, and do not necessarily reflect the views of the US Department of Defense or Federal Government. 
manifestations, and mechanism of antidepressant-associated bruxism. We present a case of SSRI-associated bruxism encountered in our neurology clinic, followed by a systematic review of similar case reports. We also include a discussion of epidemiology and proposed pathophysiology of this phenomenon. Finally, we discuss effective treatment strategies based on literature review.

\section{Case report}

A 40-year-old woman with a history of anxiety, depression, and posttraumatic stress disorder presented with persistent right-sided jaw pain and jaw opening limitations for approximately 2 years. She had seen multiple providers for these symptoms, including orofacial pain and dentistry. She described her symptoms as an achy, persistent right-sided pain, worse in the morning. Her symptoms had been so severe that she had been unable to open her mouth widely or chew food on the right side. Consequently, she had only been eating soft foods and chewing food on the left side of her mouth. She denied headaches, numbness and tingling of the face, changes in vision and hearing, loss of taste, difficulty with coordination, or numbness, tingling, and weakness in any other parts of the body.

Over the course of her workup, the patient was fitted with a mouth guard for sleep bruxism, which did not improve her symptoms. Brain MRI obtained prior to her referral to neurology was unremarkable. Her only medications were sertraline $40 \mathrm{mg}$ daily for depression and occasional cetirizine for allergic rhinitis. She had never taken dopamine-blocking therapies. Her neurologic examination at that time was notable for right-sided masseter hypertrophy and tenderness to palpation of the muscles of mastication bilaterally. She was noted to be cautious with opening her mouth for examination, and was unable to open it widely due to pain and spasm.

The patient's symptoms were initially thought to be due to idiopathic refractory temporomandibular joint disorder with a possible oromandibular dystonia given her jaw opening limitations on the right side. She was treated with a trial of onabotulinum toxin injections to the right masseter. Three months later, the injections had had no effect on her jaw pain or jaw opening limitation (which was still painful). However, she was concerned that her jaw pain may have been related to her SSRI, revealing that her symptoms started soon after she began this medicine 2 years prior. As a result, she decided to discontinue her antidepressant. Approximately 3 days after discontinuing this medicine, her jaw pain and spasm completely resolved, and she was again able to fully open her mouth. After her depression symptoms worsened, she was started on venlafaxine. She noted that her jaw symptoms returned, but were less severe than with sertraline. The patient's Adverse Drug Reaction Probability Scale score for sertraline was 9, correlating to Definite Adverse Drug Reaction. ${ }^{3}$ Upon changing her venlafaxine to bupropion, her jaw pain resolved, and her depression was adequately treated.
There is some evidence that bruxism

\section{and jaw pain may be associated with SSRI/SNRI use.}

\section{Background \\ Jaw pain associated with antidepressant use}

Jaw pain and bruxism are common, associated with a wide variety of disorders. Idiopathic bruxism is estimated to affect between 5\% and $20 \%$ of the adult population. ${ }^{1,4,5}$ Age at onset is typically between age 10 and 20 , and children tend to be affected at similar rates compared to adults. ${ }^{1,4}$ Awake bruxism is more commonly observed in women, ${ }^{4}$ where no sex preference is observed in sleep bruxism. ${ }^{1}$ Children of sleep bruxers are more likely to develop sleep bruxism than children of people who never experienced either sleep or daytime bruxism, suggesting a possible genetic predisposition. ${ }^{1}$ Bruxism can resolve spontaneously and may recur later in life, ${ }^{5}$ but is overall less commonly observed among the elderly. ${ }^{4}$

The epidemiology of antidepressant-associated bruxism and jaw pain is unknown. Commonly reported adverse effects related to SSRI use include sexual dysfunction, drowsiness, and weight gain, which occur most commonly within the first 3 months of treatment. ${ }^{6}$ Among SNRIs, nausea, dry mouth, diaphoresis, dizziness, and headache are most common. ${ }^{7}$ Bupropion, a norepinephrine-dopamine reuptake inhibitor (NDRI), is more likely to cause weight loss when compared to other antidepressants, and is less likely to cause sexual dysfunction. ${ }^{8}$ Bruxism may be an underreported side effect of these medications, as data are limited to case reports and cross-sectional studies. Data may be limited by reporting bias or limited awareness of this effect by clinicians.

There is some evidence that bruxism and jaw pain may be associated with SSRI/SNRI use. Along with several case reports, one cross-sectional study assessed rates of sleep bruxism among patients taking serotonergic antidepressants compared to controls. ${ }^{5}$ The overall prevalence of bruxism was higher in the antidepressant group compared to the control group ( $24.3 \%$ vs $15.3 \%, p=0.002)$, with highest prevalence of bruxism among patients taking paroxetine, venlafaxine, and duloxetine. This study also reported that most patients reported onset of bruxism within 4 months of starting their antidepressant, with a mean onset time of 2.85 months after starting therapy. Finally, while there was no significant relationship with sex, marital status, level of education, employment status, or smoking, mean patient age was higher among patients experiencing antidepressantassociated bruxism compared to controls who did not experience this effect ( 41.37 vs $37.48, p=0.003$ ). These data suggest that age may influence the development of bruxism in the setting of antidepressant use. Given the paucity of reports in the neurologic literature, further exploration of 
Table 1 Patient data included in literature search and analysis, by article

\begin{tabular}{|c|c|c|c|c|c|c|c|}
\hline Reference & Age, y & Sex & Offending agent & Total mg/d & $\begin{array}{l}\text { Time to symptom } \\
\text { onset, wk }\end{array}$ & Treatment & $\begin{array}{l}\text { Time to symptom } \\
\text { resolution, wk }\end{array}$ \\
\hline \multirow[t]{2}{*}{9} & NR & $N R$ & Citalopram & 20 & 6 & Dose reduction & NR \\
\hline & NR & $N R$ & Citalopram & 40 & 3 & Dose reduction & NR \\
\hline 10 & 61 & $\mathrm{~F}$ & Fluvoxamine & 100 & 3 & Cessation & 2 \\
\hline 11 & 32 & $\mathrm{~F}$ & Duloxetine & 60 & 3 & Buspirone & 2 \\
\hline \multirow[t]{2}{*}{12} & 29 & $\mathrm{~F}$ & Venlafaxine & 150 & 2 & Cessation & NR \\
\hline & 36 & $\mathrm{~F}$ & Venlafaxine & 75 & 3 & Cessation & 0.43 \\
\hline 13 & 38 & $\mathrm{~F}$ & Paroxetine & 20 & 3 & Buspirone & 2 \\
\hline 14 & 34 & M & Fluoxetine & 60 & 2 & Chlorpromazine & 4 \\
\hline \multirow[t]{2}{*}{15} & 54 & M & Escitalopram & 10 & 2 & Buspirone & NR \\
\hline & 44 & $\mathrm{~F}$ & Escitalopram & 15 & 234 & Tandospirone & 1 \\
\hline 16 & 7 & M & Fluoxetine & 15 & 4 & Cessation & 0.71 \\
\hline 17 & 8 & M & Atomoxetine & 40 & 4 & Buspirone & 1.43 \\
\hline \multirow[t]{2}{*}{18} & 24 & $\mathrm{~F}$ & Citalopram & 20 & 4 & Buspirone & 2 \\
\hline & 32 & $\mathrm{~F}$ & Paroxetine & 30 & 2 & Dose reduction & 2 \\
\hline 19 & 62 & M & Venlafaxine & 150 & 2 & Cessation & 8 \\
\hline \multirow[t]{4}{*}{20} & 35 & M & Sertraline & 100 & 2 & Buspirone & 4 \\
\hline & 61 & $\mathrm{~F}$ & Sertraline & 100 & 4 & Buspirone & 3 \\
\hline & 38 & $\mathrm{~F}$ & Sertraline & 150 & 4 & Buspirone & 3 \\
\hline & 32 & $\mathrm{~F}$ & Sertraline & 100 & 1 & Buspirone & 8 \\
\hline 21 & 44 & M & Bupropion SR & 300 & 0.29 & Dose reduction & NR \\
\hline \multirow[t]{4}{*}{22} & 36 & $\mathrm{~F}$ & Fluoxetine & 20 & 4 & Dose reduction & NR \\
\hline & 43 & $\mathrm{~F}$ & Fluoxetine & 15 & 2 & Buspirone & NR \\
\hline & 30 & $\mathrm{~F}$ & Fluoxetine & 20 & 2 & Buspirone & 1 \\
\hline & 36 & $\mathrm{~F}$ & Sertraline & 25 & 2 & Buspirone & 1 \\
\hline \multirow[t]{6}{*}{23} & 73 & $\mathrm{~F}$ & Sertraline & 100 & 2 & Dose reduction & NR \\
\hline & 28 & $\mathrm{~F}$ & Fluoxetine & 20 & 1 & Buspirone & 1 \\
\hline & 67 & $\mathrm{~F}$ & Paroxetine & 20 & 44 & Chlorpromazine & NR \\
\hline & 41 & $\mathrm{~F}$ & Fluoxetine & 20 & 1 & Cessation & 0.14 \\
\hline & 30 & $\mathrm{~F}$ & Fluoxetine & 20 & 20 & Cessation & 4 \\
\hline & 61 & M & Fluoxetine & 20 & 12 & Cessation & 72 \\
\hline 24 & 34 & M & Escitalopram & 20 & NR & Buspirone & 1.43 \\
\hline 25 & 35 & M & Venlafaxine & 150 & 1 & Cessation & 0.29 \\
\hline 26 & 81 & M & Paroxetine & 30 & 6 & Cessation & 1 \\
\hline 27 & 31 & $\mathrm{~F}$ & Venlafaxine & 150 & 1 & Trazodone & 1.43 \\
\hline 28 & 43 & $\mathrm{~F}$ & Paroxetine & 20 & NR & No change & NR \\
\hline 29 & 12 & M & Atomoxetine & 10 & 4 & Buspirone & 12 \\
\hline 30 & 45 & M & Escitalopram & 20 & 3 & Tandospirone & 3 \\
\hline
\end{tabular}


Table 1 (Continued)

\begin{tabular}{|c|c|c|c|c|c|c|c|}
\hline Reference & Age, y & Sex & Offending agent & Total mg/d & $\begin{array}{l}\text { Time to symptom } \\
\text { onset, wk }\end{array}$ & Treatment & $\begin{array}{l}\text { Time to symptom } \\
\text { resolution, wk }\end{array}$ \\
\hline 31 & 44 & $\mathrm{~F}$ & Duloxetine & 60 & 3 & Buspirone & 0.57 \\
\hline 32 & 63 & $\mathrm{~F}$ & Fluoxetine & 60 & 6 & Cessation & 1 \\
\hline 33 & 64 & $\mathrm{~F}$ & Venlafaxine & 150 & 6 & Buspirone & 4 \\
\hline \multirow[t]{2}{*}{34} & 48 & M & Escitalopram & 40 & 4 & Buspirone & 4 \\
\hline & 18 & $\mathrm{~F}$ & Venlafaxine & 225 & 3 & Amitriptyline & 0.43 \\
\hline 35 & 20 & $\mathrm{~F}$ & Paroxetine & 20 & 8 & Aripiprazole & 0.57 \\
\hline 36 & 15 & $\mathrm{~F}$ & Fluoxetine & 20 & NR & Buspirone & 1 \\
\hline 37 & 43 & $\mathrm{~F}$ & Fluoxetine & 20 & 16 & Gabapentin & 0.71 \\
\hline Author case report & 40 & $\mathrm{~F}$ & Sertraline & 40 & NR & Dose reduction & 0.43 \\
\hline
\end{tabular}

Abbreviation: $\mathrm{NR}=$ not reported.

this topic may provide increased awareness among neurologists about the existence of this condition and effective management strategies. The objective of this article was to review the existing literature for the clinical features of antidepressantassociated bruxism, to identify common offending agents, and to explore successful treatment strategies.

\section{Systematic review}

\section{Data sources and data extraction}

We searched PubMed for case reports and case series relating to antidepressant-associated bruxism using the following search terms: "SSRI bruxism," "SNRI bruxism," "citalopram bruxism," "escitalopram bruxism," "buspirone bruxism," and "antidepressant bruxism." Search was not limited by publication date, and we applied no language restrictions. Case reports, case series, and letters to the editor containing reports of at least one case of suspected antidepressant-associated bruxism were included. Articles were excluded if they did not include information regarding symptom management. We identified additional reports from the reference lists of retrieved reports and from reviews found during literature search. We recorded demographic information, psychiatric diagnoses, time to symptom onset, offending agents, interventions, and time to symptom resolution for inclusion in our systematic review. The purpose of this systematic review was to compare clinical features and effective treatment interventions of antidepressant-associated bruxism using patient data from published case reports.

\section{Results}

Literature search initially returned 67 unique articles, with 4 additional articles identified using references in articles above, for a total of 71 articles reviewed for inclusion. Of the 71 total articles reviewed, 42 were excluded for failing to meet inclusion criteria, and a total of 29 articles were included, containing a total of 45 unique cases. ${ }^{9-37}$ Basic demographic data (age and sex) for each patient were included in all but 1 article, which documented offending agent and treatment, but did not include sex or age of the 2 patients presented. Data from the case presented above were added to the total number of cases and included in data analysis (tables 1 and 2).

A total of 46 patients were included in this analysis. The average age was 39.8 years, with an age range of $7-81$ years. Thirty of the 44 reported patients were female $(68 \%)$. Most patients $(76 \%)$ were being treated for a diagnosis of major depressive disorder, generalized anxiety disorder, or a combination of these conditions (data not shown below). Other diagnoses included bipolar II, attention-deficit/hyperactivity disorder, somatoform disorder, fibromyalgia, obsessivecompulsive disorder, and complicated grief.

A total of 10 offending agents were identified. Six SSRIs were reported (citalopram, escitalopram, fluoxetine, fluvoxamine, paroxetine, and sertraline), along with 3 SNRIs (atomoxetine, duloxetine, and venlafaxine), and 1 NDRI (bupropion). The majority of agents reported were SSRIs (74\%), followed by SNRIs (24\%). The most commonly reported agents were fluoxetine (12 cases), followed by venlafaxine and sertraline ( 7 cases each).

A total of 11 beneficial interventions were reported. The most commonly reported intervention was the addition of buspirone to the patient's current regimen (reported in 20 cases), followed by cessation of the medicine ( 12 cases) and dose reduction ( 7 cases). One article reported successful use of electroconvulsive therapy in alleviating symptoms after 2 weeks of adjunctive tandospirone was ineffective. ${ }^{10}$ Three articles reported alleviation of symptoms with cessation of the offending agent in combination with buspirone therapy. ${ }^{11-13}$ One case reported spontaneous resolution of symptoms 
Table 2 Patient data

\begin{tabular}{|c|c|}
\hline & Values, n (\%) \\
\hline \multicolumn{2}{|l|}{ Demographics } \\
\hline Total patients & $46(100)$ \\
\hline Female sex; 2 did not report & $30(68)$ \\
\hline Age range, y (average) & $7-81(39.8)$ \\
\hline \multicolumn{2}{|l|}{ Offending agents } \\
\hline Selective serotonin reuptake inhibitors & $34(74)$ \\
\hline Citalopram & $3(7)$ \\
\hline Escitalopram & $5(11)$ \\
\hline Fluoxetine & $12(26)$ \\
\hline Fluvoxamine & $1(2)$ \\
\hline Paroxetine & $6(13)$ \\
\hline Sertraline & $7(15)$ \\
\hline $\begin{array}{l}\text { Serotonin-norepinephrine reuptake } \\
\text { inhibitors }\end{array}$ & $11(24)$ \\
\hline Atomoxetine & $2(4)$ \\
\hline Duloxetine & $2(4)$ \\
\hline Venlafaxine & $7(15)$ \\
\hline Other & $1(2)$ \\
\hline Bupropion & $1(2)$ \\
\hline \multicolumn{2}{|l|}{ Interventions } \\
\hline \multicolumn{2}{|l|}{ Pharmacologic } \\
\hline Amitriptyline & $1(2)$ \\
\hline Aripiprazole & $1(2)$ \\
\hline Buspirone & $19(41)$ \\
\hline Cessation & $12(26)$ \\
\hline Chlorpromazine & $2(4)$ \\
\hline Dose reduction & $7(15)$ \\
\hline Gabapentin & $1(2)$ \\
\hline No change & $1(2)$ \\
\hline Tandospirone & $1(2)$ \\
\hline Trazodone & $1(2)$ \\
\hline \multicolumn{2}{|l|}{ Other } \\
\hline Electroconvulsive therapy & $1(2)$ \\
\hline
\end{tabular}

without any interventions. ${ }^{14}$ In the above articles, buspirone dosage typically ranged between 5 and $30 \mathrm{mg}$ administered in $1-3$ doses per day.

Time to onset of symptoms, in most cases, was between 3 and 4 weeks. One case reported bruxism of many years that was attributed to antidepressant use only after discontinuation of the medication resulted in rapid cessation of symptoms. ${ }^{15}$ Most patients reported cessation of symptoms, from the time of last intervention, between 2 and 3 weeks. The case of spontaneous symptom resolution without intervention, as mentioned, reported resolution after 4 weeks. ${ }^{14}$

\section{Discussion}

The literature suggests an association with serotonergic antidepressants inducing jaw pain/jaw spasm reversible syndrome. Data from the pooled case reports above suggest that antidepressant-associated bruxism may occur in patients of any age, including children. ${ }^{16,17}$ Symptoms tend to appear within 3-4 weeks of beginning the antidepressant medicine or undergoing dose titration. Symptom resolution may be achieved through the addition of serotonin $1 \mathrm{~A}\left(5 \mathrm{HT}_{1 \mathrm{~A}}\right)$ partial agonists (buspirone, tandospirone), by dose reduction, by medicine cessation, or by the addition of other pharmacologic agents, including tricyclic antidepressants (amitriptyline), antipsychotics (aripiprazole, chlorpromazine), norepinephrine-dopamine reuptake inhibitors (bupropion), or serotonin $2 \mathrm{~A} / 2 \mathrm{C}$ antagonist and reuptake inhibitors (trazodone). Symptoms may also resolve over time without pharmacologic intervention.

The available literature suggests a strategy for treatment of patients with SSRI/SNRI-associated jaw pain. Several cases reported success in alleviating symptoms with the addition of buspirone. This observation can be instructive in both understanding the underlying pathophysiologic mechanisms of antidepressant-associated bruxism, as well as in providing a foundation for treatment recommendations. First, buspirone is a serotonin $1 \mathrm{~A}\left(5 \mathrm{HT}_{1 \mathrm{~A}}\right)$ partial agonist at both presynaptic and postsynaptic receptors at various sites, including the amygdala, prefrontal cortex, thalamus, and striatum. ${ }^{38}$ Buspirone is commonly used as a generalized anxiolytic, but can also be used to augment SSRIs/ SNRIs, by combining its $5 \mathrm{HT}_{1 \mathrm{~A}}$ partial agonism with the serotonin transporter inhibition achieved by the SSRI/ SNRI. It is also believed that buspirone may work via adaptive neuronal and receptor mechanisms, instead of acute receptor occupancy, which may explain the observed 2- to 3-week latency in alleviating bruxism associated with serotonergic antidepressants. This may also explain why buspirone is ineffective in treating idiopathic bruxism. Therefore, buspirone may help to alleviate antidepressantassociated bruxism through its partial agonism of striatal $5 \mathrm{HT}_{1 \mathrm{~A}}$ receptors. Interestingly, aripiprazole is also a $5 \mathrm{HT}_{1 \mathrm{~A}}$ partial agonist throughout the brain, and may work through a similar mechanism to alleviate antidepressant-associated bruxism. $^{38}$

The above data suggest that the addition of buspirone 5-10 $\mathrm{mg}$ up to 3 times daily may be an effective first-line option for alleviating antidepressant-associated bruxism, particularly in 
Bruxism may develop as an adverse reaction to antidepressant therapy, and is most likely to develop within 2-3 weeks of medication introduction or dose titration.

patients who may not tolerate dose reduction or medication cessation. If the patient can tolerate dose reduction or drug discontinuation, this may likewise be a reasonable course of action. Finally, in patients who are not experiencing severe symptoms and who are satisfied with antidepressant effects otherwise, simple monitoring for spontaneous resolution of symptoms may be considered.

We recognize the limitations of this systematic review. Principally, the analysis above is based on a collection of case reports, and does not represent randomized or placebocontrolled study data. Publication bias limits the true number of cases available in the literature for review, and therefore other offending agents or treatments may have been observed by others that are not represented here. Finally, further prospective study would be helpful to elucidate the true underlying effect of the interventions described above compared to watchful waiting for symptom resolution.

Bruxism may develop as an adverse reaction to antidepressant therapy, and is most likely to develop within 2-3 weeks of medication introduction or dose titration. This phenomenon may be seen in a variety of serotonergic antidepressants, and may be most associated with fluoxetine, sertraline, or venlafaxine. Patients who experience this condition may benefit from the addition of buspirone 5 and 10 $\mathrm{mg}$ in daily, twice daily, or 3 times daily dosing; dose reduction and antidepressant cessation may also be considered. Antidepressant-associated bruxism may be an underreported condition, particularly in the neurology clinic. Further prospective trials may help to elucidate optimal therapies for this condition.

\section{Author contributions}

A. Garrett: study concept and design, acquisition of data, analysis and interpretation of data. J. Hawley: study supervision, critical revision of manuscript for intellectual content.

\section{Study funding}

No targeted funding reported.

\section{Disclosure}

The authors report no disclosures. Full disclosure form information provided by the authors is available with the full text of this article at Neurology.org/cp.
TAKE-HOME POINTS

$\rightarrow$ Bruxism associated with antidepressant use is an underrecognized phenomenon, particularly among neurologists.

$\rightarrow$ Antidepressant-associated bruxism/jaw pain most commonly begins within 3-4 weeks of medication initiation or dose titration, and can resolve within 3-4 weeks of drug discontinuation.

$\rightarrow$ Patients commonly present with jaw pain, teeth grinding, and trismus in the setting of drugs like fluoxetine, sertraline, and venlafaxine.

$\rightarrow$ Besides drug discontinuation, alternative treatments for drug-induced bruxism include the addition of buspirone or the substitution of other pharmacologic agents.

Received August 25, 2017. Accepted in final form December 27, 2017.

\section{References}

1. American Academy of Sleep Medicine. The International Classification of Sleep Disorders: Diagnostic and Coding Manual. 3rd ed. Darien, IL: American Academy of Sleep Medicine; 2014.

2. McAuliffe P. Types of bruxism. J Ir Dent Assoc 2012;58:138-141.

3. Naranjo CA, Busto U, Sellers EM, et al. A method for estimating the probability of adverse drug reactions. Clin Pharmacol Ther 1981;30:239-245.

4. Shetty S, Pitti V, Satish Babu CL, Surendra Kumar GP, Deepthi BC. Bruxism: a literature review. J Indian Prosthodont Soc 2010;10:141-148.

5. Uca AU, Uğuz F, Kozak HH, et al. Antidepressant-induced sleep bruxism: prevalence, incidence, and related factors. Clin Neuropharmacol 2015;38:227-230.

6. $\mathrm{Hu} \mathrm{XH}, \mathrm{Bull} \mathrm{SA}$, Hunkeler EM, et al. Incidence and duration of side effects and those rated as bothersome with selective serotonin reuptake inhibitor treatmen for depression: patient report versus physician estimate. J Clin Psychiatry 2004; 65:959-965.

7. Mental health medications [online]. National Institutes of Mental Health; 2016 Available at: nimh.nih.gov/health/topics/mental-health-medications/index.shtml. Accessed August 1, 2017.

8. Patel K, Allen S, Haque MN, Angelescu I, Baumeister D, Tracy DK. Bupropion: a systematic review and meta-analysis of effectiveness as an antidepressant. Ther Adv Psychopharmacol 2016;6:99-144.

9. Wise MEJ. Citalopram-induced bruxism. Br J Psychiatry 2001;178:182.

10. Miyaoka T, Yasukawa R, Mihara T, et al. Successful electroconvulsive therapy in major depression with fluvoxamine-induced bruxism. J ECT 2003;19:170-172.

11. Albayrak Y, Ekinci O. Duloxetine-induced nocturnal bruxism resolved by buspirone: case report. Clin Neuropharmacol 2011;34:137-138.

12. Jaffee MS, Bostwick JM. Buspirone as an antidote to venlafaxine-induced bruxism. Psychosomatics 2000;41:535-536.

13. Milanlioglu A. Paroxetine-induced severe sleep bruxism successfully treated with buspirone. Clinics 2012;67:191-192.

14. Iskandar JW, Wood B, Ali R, Wood RL. Successful monitoring of fluoxetine-induced nocturnal bruxism: a case report. J Clin Psychiatry 2012;73:366.

15. Raja M, Raja S. Two cases of sleep bruxism associated with escitalopram treatment. J Clin Psychopharmacol 2014;34:403-405.

16. Çolak Sivri R, Akça ÖF. Buspirone in the treatment of fluoxetine-induced sleep bruxism. J Child Adolesc Psychopharmacol 2016;26:762-763.

17. Yüce M, Karabekiroğlu K, Say GN, Müjdeci M, Oran M. Buspirone use in the treatment of atomoxetine-induced bruxism. J Child Adolesc Psychopharmacol 2013; 23:634-635.

18. Ak M, Gulsun M, Uzun O, Gumus HO. Bruxism associated with serotonin reuptake inhibitors: two cases. J Clin Psychopharmacol 2009;29:620-622.

19. Alonso-Navarro H, Martín-Prieto M, Ruiz-Ezquerro JJ, Jiménez-Jiménez FJ. Bruxism possibly induced by venlafaxine. Clin Neuropharmacol 2009;32:111-112.

20. Bostwick JM, Jaffee MS. Buspirone as an antidote to SSRI-induced bruxism in 4 cases. J Clin Psychiatry 1999;60:857-860.

21. Detweiler MB, Harpold GJ. Bupropion-induced acute dystonia. Ann Pharmacothe 2002;36:251-254.

22. Ellison JM, Stanziani P. SSRI-associated nocturnal bruxism in four patients. J Clin Psychiatry 1993;54:432-434. 
23. Fitzgerald K, Healy D. Dystonias and dyskinesias of the jaw associated with the use of SSRIs. Hum Psychopharmacol 1995;10:215-219.

24. Grinshpoon A, Weizman A, Amrami-Weizman A. The beneficial effect of trazodone treatment on escitalopram-associated nocturnal bruxism. J Clin Psychopharmacol 2014;34:662

25. Jose SP. Venlafaxine-induced severe sleep bruxism in a patient with generalized anxiety disorder. Indian J Psychol Med 2015;37:249-250.

26. Kishi Y. Paroxetine-induced bruxism effectively treated with tandospirone. J Neuropsychiatry Clin Neurosci 2007;19:90-91.

27. Kuloglu M, Ekinci O, Caykoylu A. Venlafaxine-associated nocturnal bruxism in a depressive patient successfully treated with buspirone. J Psychopharmacol Oxf Engl 2010;24:627-628.

28. Lobbezoo F, van Denderen RJ, Verheij JG, Naeije M. Reports of SSRI-associated bruxism in the family physician's office. J Orofac Pain 2001;15:340-346.

29. Mendhekar D, Lohia D. Worsening of bruxism with atomoxetine: a case report. World J Biol Psychiatry 2009;10:671-672.

30. Mukherjee S, Sen S, Biswas A, Chatterjee SS, Tripathi SK. Escitalopram induced bruxism: a case report. Sch J Appl Med Sci 2014;2:1162-1163.
31. Şahin Onat S, Malas FÜ. Duloxetine-induced sleep bruxism in fibromyalgia successfully treated with amitriptyline. Acta Reumatol Port 2015;40:391-392.

32. Oulis P, Dimitrakopoulos S, Konstantakopoulos G, Tsaltas E, Kollias K. Low-dose aripiprazole in the treatment of SSRI-induced bruxism. J Neuropsychiatry Clin Neurosci 2012;24:E39.

33. Pavlovic ZM. Buspirone to improve compliance in venlafaxine-induced movement disorder. Int J Neuropsychopharmacol 2004;7:523-524.

34. Ranjan S. S chandra P, Prabhu S. Antidepressant-induced bruxism: need for buspirone? Int J Neuropsychopharmacol 2006;9:485-487.

35. Romanelli F, Adler DA, Bungay KM. Possible paroxetine-induced bruxism. Ann Pharmacother 1996;30:1246-1248.

36. Sabuncuoglu O, Ekinci O, Berkem M. Fluoxetine-induced sleep bruxism in an adolescent treated with buspirone: a case report. Spec Care Dentist 2009;29:215-217.

37. Soyata AZ, Oflaz S. Gabapentin treatment in bruxism associated with fluoxetine. J Clin Psychopharmacol 2015;35:481-482.

38. Stahl SM. Stahl's Essential Psychopharmacology: Neuroscientific Basis and Practical Applications. 4th ed. Cambridge: Cambridge University Press; 2013.

\section{Share Your Insights, Expertise, and Experiences}

- How are you employing drugs and devices in your field?

- What ethical challenges do you face?

- Do you have a case report that is illustrative of a clinical challenge?

- What challenges have you faced or successes have you enjoyed in bringing greater efficiency to your practice?

Deliver a high-quality, peer-reviewed message to your colleagues in practice, submit your paper at NPub.org/NCP/submit. 